Sind die im 20. Jahrhundert gebauten urbanen Systeme überlebensfähig? Globale und regionale Analysen zum Ressourcenhaushalt stützen folgende These:

Der Weg zu einer "nachhaltigen Lebensform menschlicher Gesellschaften" führt in den nächsten Jahrzehnten über den Umbau der bereits gebauten urbanen Systeme.

Dabei kommt den Wohngebăuden eine Schlüsselstellung zu. Eine erste

mathematisch-naturwissenschaftliche Modellierung von Umbauszenarios

zeigt Möglichkeiten und Grenzen bei der Anwendung sehr allgemein formulierter

Nachhailtigkeitskriterien. Der Schlüssel zur Realisierung von urbanen Umbauprozessen

liegt jedoch im regionalpolitischen Willen, eine neue urbane Identität zu schaffen.

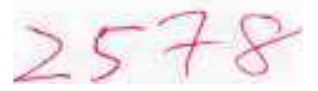

\title{
Stadt mit wenig Energie, viel Kies und neuer Identität
}

\section{Metabolische Modelle für den Umbau urbaner Siedlungen am Beispiel der Wohngebäude}

\author{
Michael Redle und Peter Baccini*
}

\section{Stadtentwicklung und nachhaltiger Ressourcenhaushalt}

Die siedlungsgeographischen Analysen der letzten Jahrzehnte und die auf demographischen Daten basierenden Prognosen für die nächsten Dekaden weisen weltweit auf eine Verschiebung der Wohnbevölkerung vom ruralen in den urbanen Raum. In den entwickelten Ländern lebt schon heute die große Mehrheit in städtischen Siedlungen. Global betrachtet fällt auf, $\mathrm{da} ß$ in diesen Gebieten während der letzten 40 Jahre die Siedlungsfläche verdoppelt wurde, und dies nicht primär wegen einer rapid steigenden Bevölkerungszahl, sondern weil der Bedarf pro Kopf exponentiell zugenommen hat. Damit verbunden sind auch stark angeschwollene Energie-, Wasser- und Baumaterialflüsse.

In den politischen Programmen zur Umweltgestaltung und zur Ressourcennutzung wird immer häufiger der Be-

\footnotetext{
* Postadresse : Prof. Dr. P. Baccini

Professur für Stoffhaushalt und

Entsorgungstechnik

Departement Bau und Umwelt

Eidgenössische Technische Hochschule Zürich

EAWAG

CH-8600 Dübendorf (Schweiz)

E-Mail: baccini@eawag.ch
}

griff "nachhaltige Entwicklung" oder kurz "Nachhaltigkeit" verwendet. Die kürzeste Definition dieses Begriffs findet sich im sogenannten BrundtlandBericht (WECD 1987): Die Bedürfnisse der Gegenwart befriedigen, ohne zu riskieren, daß künftige Generationen ihre eigenen Bedürfnisse nicht befriedigen können. Es handelt sich um eine global orientierte und ethisch motivierte Zielvorgabe. Sie stellt uns vor eine lange Reihe von schwierigen Fragen konzeptioneller und methodischer Art. Wie soll man im aktuellen Aktionsfeld menschlicher Tätigkeiten, also auf der Stufe Regionen, im Bereich von Wirtschaftsbranchen, der öffentlichen Hand und in den Privathaushalten, konkrete Programme zu dieser allgemeinen Zielvorgabe aufbauen und durchsetzen? Der Anspruch der "Nachhaltigkeit" umfaßt sowohl die geogenen Systeme (sogenannte natürliche Systeme wie Ozeane und tropische Regenwälder) als auch die anthropogenen Systeme (zum Beispiel landwirtschaftliche Böden, Transportnetze für Personen und Güter in Siedlungsgebieten). Für die anthropogenen Systeme gilt zudem, daß "Nachhaltigkeit" nicht nur die langfristige Sicherung ökologischer Qualitäten bedeutet, sondern auch jene der wirtschaftlichen, sozialen und kulturellen Leistungen.
In diesem Beitrag sollen die naturwissenschaftlich-ökologischen Anforderungen der Nachhaltigkeit an einem konkreten Beispiel erörtert werden. Diese Anforderungen können in drei Punkten zusammengefaßt werden (ebenfalls unter Rückgriff auf den BrundtlandBericht):

- Durch unser Handeln keine "übernatürlich" große Rate von Artensterben induzieren (Bewahrung des "genetischen Kapitals aus der Evolution").

- Keine Ressourcen schneller verbrauchen, als diese natürlich entstehen können.

- Keine "Altlasten" (zum Beispiel Deponien, verschmutzte Ökosysteme) hinterlassen, welche die Bewegungsfreiheit unserer Nachkommen einschränken.

Erste Studien über den Ressourcenhaushalt dicht besiedelter Regionen kommen zum Schluß (so Buitenkamp et al. für die Niederlande ${ }^{[1]}$ ), daß deren aktuelle Ressourcenwirtschaft den obengenannten drei Kriterien nicht genügt. Betrachtet man den Metabolismus solcher Regionen differenziert [2], so wird deutlich, daß die Aktivitäten Wohnen und Arbeiten sowie Transportieren und Kommunizieren für 80-90 Prozent der Materielager und der Energie- und Materieflüsse maßgeblich sind. Auch kann gezeigt werden, daß der geschwindigkeitsbestimmende Schritt des regio- 
nalen Metabolismus durch die Veränderungsrate der Gebäude und der Transportnetze bestimmt wird ${ }^{[3]}$.

\section{Umbauszenarios für die Aktivität Wohnen}

Für "Wohnen" als Aktivität in urbanen Siedlungen lassen sich zwei wesentliche Faktoren hervorheben ${ }^{[4]}$. Erstens: Die Siedlungsstruktur (Gebäude, Plätze, Straßen et cetera) schafft die Rahmenbedingungen für die Wahrnehmung der Stadt, das Zusammenleben ihrer Bewohner und den Ressourcenverbrauch. Zweitens: Die Bewohner der Stadt entscheiden, wie sie diese Struktur nutzen, und bestimmen so das konkrete Straßenbild, das soziale Leben und die Ressourceneffizienz wesentlich mit. Durch den Umbau der Siedlungsstruktur sollen Rahmenbedingungen geschaffen werden, unter denen die Bewohner die Ressourcen effizienter nutzen und eine größere Vielfalt von Wohn- und Lebenssituationen erleben (zum Beispiel durch soziale Durchmischung). Diese Rahmenbedingungen sollen außerdem eine flexible Anpassung der Raumnutzung und -gestaltung an Veränderungen ermöglichen.

Wohngebäude, welche die gebaute "Haut" der Privathaushalte einer Gesellschaft darstellen, bilden die Kernstücke jeder Siedlung. Die Umsetzung allgemeiner Zielvorstellungen zur "nachhaltigen Entwicklung" verlangt auch konkrete Vorgaben für den Einsatz der wichtigsten materiellen Ressourcen in der künftigen Gestaltung von Wohngebäuden. Es sind dies die Gesteine, die Energieträger und die bereits vorhandenen Ressourcenlager in den Gebäuden. Mathematisch-naturwissenschaftliche Modelle ${ }^{[2]}$ dienen dazu, die Abhängigkeiten dieser drei Ressourcengruppen in unterschiedlichen Entwicklungsszenarios aufzuzeigen.

Hier richten wir den Blick nun ausschließlich auf die metabolischen Schlüsselgrößen der Aktivität Wohnen, nämlich auf die Energieträger und das quantitativ wichtigste Baumaterial, den Kies beziehungsweise daraus hergestellten Beton. Am Beispiel der Wohngebäude einer dicht besiedelten Region ${ }^{[5]}$ sollen zwei grundlegende Fragen beantwortet werden:

- Welche stoffliche Geschichte steckt im bestehenden Lager von Wohngebäuden, und welchen Energiefluß bewirken deren Bau und Betrieb?
- Welche materiellen und energetischen Szenarios für die Veränderung von Wohngebäuden sind vorstellbar, um dem Ziel einer nachhaltigen Qualität nahezukommen?

Die Qualität der "Nachhaltigkeit" läßt sich für diesen Fall auf das zweite der in Abschnitt 1 genannten drei Kriterien beschränken, also auf den Einsatz von erneuerbaren Ressourcen (Baumaterialien und Energieträger).

\section{Methoden zur Untersuchung des Metabolismus}

Zur Beantwortung der beiden zentralen Fragen benutzen wir die Methode der Stoffflußanalyse ${ }^{[2]}$. Es geht darum, für den Aufbau, Betrieb und Abbau von Wohngebäuden in einer definierten Region ein möglichst einfaches metabolisches System derart einzurichten, $\mathrm{da} B$ die wichtigsten naturwissenschaftlichen und technischen Eigenschaften darin Platz finden und die Auswirkungen ihrer Veränderungen quantifiziert werden können.

\subsection{Systemanalyse: Abstraktion des Metabolismus von Wohngebäuden}

Hinter dem gewählten System "Ressourcenhaushalt von Wohngebäuden" (Figur 1) steckt der Gedanke, daß die Entwicklung der Wohngebäude, aus der Gegenwart betrachtet, als ein Ablöseprozeß verstanden werden kann: Die schon bestehenden Gebäude können nur noch abgebrochen werden oder allenfalls noch saniert und dann später abgebrochen werden. Neue Gebäude kommen hinzu, entweder als Ersatz für die oder zusätzlich zu den bestehenden "alten" Gebäuden. Im Unterschied zu einer zirkulären Vorstellung, bei der die Gebäudeentwicklung als Kreislauf von Neubau und Abbau verstanden wird, betont diese Betrachtung einen Wechsel von Altem zu Neuem und ist daher prädestiniert, ebenjene Umbauvorgänge zu erfassen, bei denen sich neue Wohngebäude grundsätzlich von alten unterscheiden.

Das System besteht aus sechs Prozessen, die aus den aufgezählten Gründen ausgewählt wurden:

(1) "Energieverteilung": Dieser Prozeß dient dazu, die dem Gesamtsystem zugeführte Energie $T_{91}(t)$ (fossile Energieträger, Holz, Elektrizität) auf den Betrieb der Wohngebäude $\left(T_{13}(t), T_{14}(t)\right.$, $\left.T_{15}(t)\right)$ und ihre Herstellung $\left(T_{12}^{14}(t)\right)$ zu verteilen. Es wird einfachheitshalber nur mit den Energieinhalten der Energieträger gerechnet (in Megajoule pro Einwohner und Jahr), die Materieflüsse der Energieträger bleiben ausgeklammert. Ebenfalls wird nur mit dem Endenergieverbrauch gerechnet, da die Verluste bei der Bereitstellung der (vor allem fossilen) Energieträger gering sind.

(2) "Herstellung": Hierdurch werden Baumaterialien hergestellt, transportiert und zu Wohngebäuden zusammengefügt. Es werden wiederum nur die für den Massenbaustoff Beton relevanten Materieflüsse betrachtet. Vereinfachend wird auch der im Beton zu 15 Prozent als Bindemittel eingesetzte Zement weggelassen, so daß letztendlich aus Kiesgruben (Annahme: sie liegen außerhalb der Systemgrenzen) eingeführter Sand und Kies $\left(A_{72}(t)\right)$ und der durch Rückbau gewonnene Recyclingkies $\left(A_{62}(t)\right)$ zu Beton für Neubauten $\left(A_{25}(t)\right)$ verarbeitet werden. Hingegen sind beim Energieaufwand $\left(T_{12}(t)\right)$ für Herstellung und Transport der Baumaterialien auch solche Baumaterialien einbegriffen, deren Materieflüsse hier nicht berücksichtigt werden, wie Zement, Baustahl, Aluminium, Glas, Holz und so weiter. Die Energie, die bei der Herstellung der Baumaterialien in chemischer Form gespeichert wurde, wird hier vereinfachend der Abwärme $\left(T_{210}(t)\right)$ zugeschlagen, da davon ausgegangen werden kann, daß diese Energie nicht mehr zurückgewonnen wird.

(3) "Alte Wohngebäude": In diesem Prozeß befinden sich die bis zum Zeitpunkt $t_{0}$ gebauten, bestehenden Wohngebäude. Sie werden mit Energie beheizt, und das in ihnen enthaltene Baumaterial wird "gelagert".

Unter Wohngebäuden werden die in der Volkszählung der Schweiz als solche definierten Gebäude verstanden ${ }^{[6]}$. Sie machen rund die Hälfte des gesamten Gebäudeparks der Schweiz aus (sowohl nach Einheiten als auch nach Bauvolumen) ${ }^{[7]}$. In diesem Prozeß (3) und in den Prozessen (4) und (5) spielt sich die eigentliche Nutzung der Wohngebäude $\mathrm{ab}$, nämlich das Wohnen. Als Maß für den Nutzen - quasi als Gegenwert zu den Material- und Energieaufwendungen - wird im weiteren das Wohnvolumen verstanden (von den Außenflächen der Gebäude umschlossener Raum).

Als quantitativ bedeutendstes - und aus der hier untersuchten Ressource Kies bestehendes - Baumaterial wird in diesem System vereinfachend nur der Beton betrachtet. Wohnbauten der Nachkriegszeit, die in der Schweiz rund zwei 


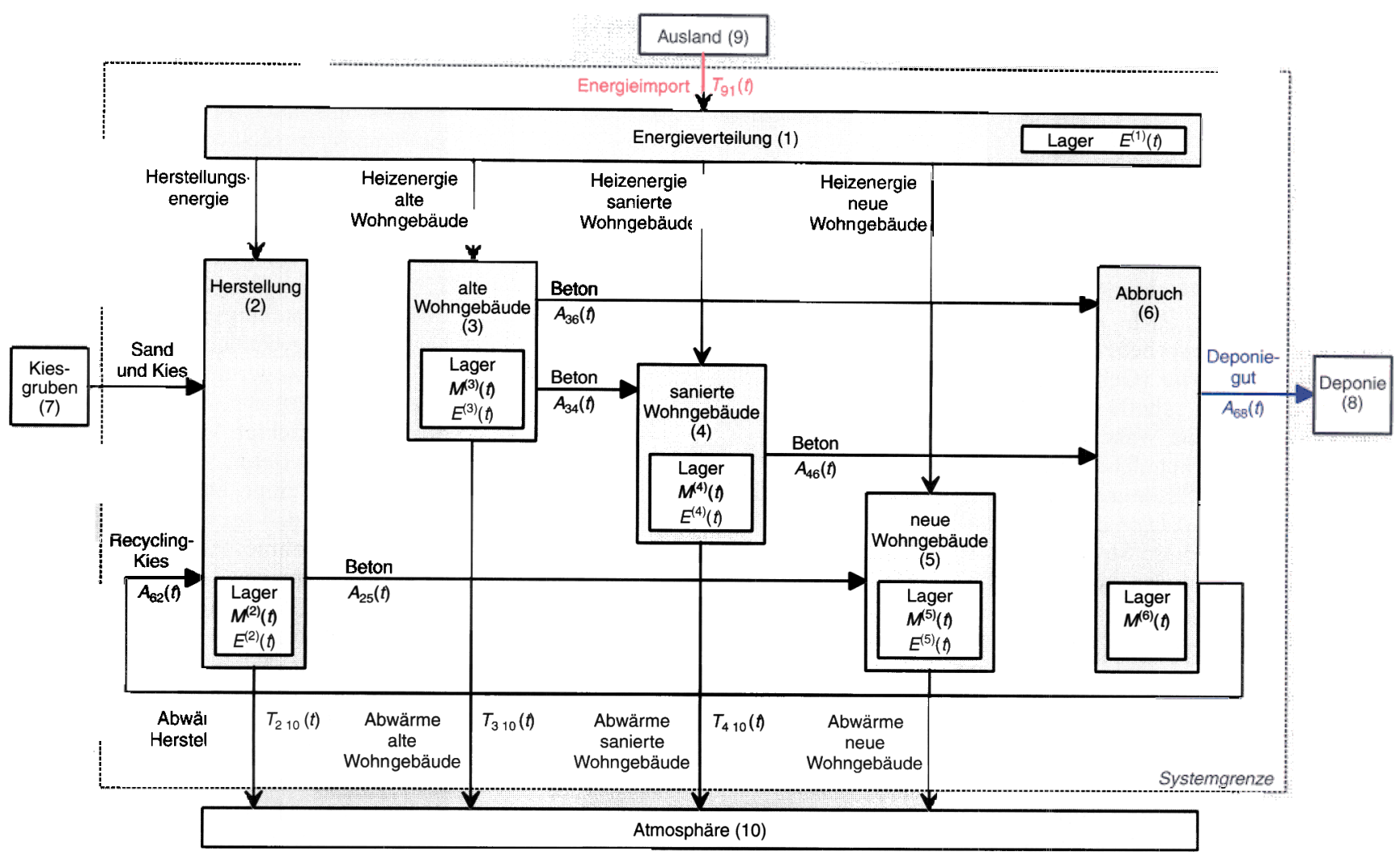

Figur 1. Das System "Ressourcenhaushalt von Wohngebäuden": Die Aufteilung des Wohngebäudeparks in alte, sanierte und neue Wohngebäude bildet eine evolutionäre Vorstellung der Entwicklung von Wohngebäuden ab. So läßt sich der Metabolismus von Umbauprozessen untersuchen. Materieflüsse $A(t)(\longrightarrow)$ sind horizontal eingezeichnet, Energieflüsse $T(t)(\longrightarrow)$ vertikal. Materielager sind mit $M(t)$ bezeichnet, Energielager mit $E(t)$.

Drittel des heutigen Wohnvolumens stellen, enthalten zwischen 50 und 90 Gewichtsprozent Beton ${ }^{[2]}$. Die Materielager der Wohngebäude $\left(M^{(3)}(t)\right)$ sind also gewissermaßen als Betongerüste oder als Rohbau der Gebäude anzusehen. Der in den alten Gebäuden befindliche Beton wird im Fall einer Sanierung auf die "sanierten Wohngebäude" übertragen $\left(A_{34}(t)\right)$, oder er wird dem "Abbruch" zugeführt $\left(A_{36}(t)\right)$.

Es wird hier nur die für die Raumheizung und Warmwassererzeugung benötigte Wärmeenergie berücksichtigt. Die für Licht, Kraft, Kochen und Geräte eingesetzte Energie wird einfachheitshalber ausgeklammert; sie ist in erster Näherung vernachlässigbar. Es wird angenommen, daß alle Energie in $\mathrm{Ab}$ wärme umgewandelt wird $\left(T_{310}(t)\right)$.

(4) "Sanierte Wohngebäude" sind jene Wohngebäude, die eine energetische Sanierung erfahren haben. Auch ihnen wird Energie zu Heizzwecken zugeführt $\left(T_{14}(t)\right)$, und Materie wird dort wieder in Form von Beton gelagert $\left(M^{(4)}(t)\right)$. Indem alte Wohngebäude energetisch modernisiert und somit zu sanierten Wohngebäuden werden, wird dem Prozeß Beton zugeführt $\left(A_{34}(t)\right)$, oder es wird ihm durch Abbruch schon sanierter Gebäude andererseits Beton entzogen $\left(A_{46}(t)\right)$. Es wird angenommen, $\mathrm{da} B$ die durch eine energetische Sanierung erfolgten Änderungen am "Betongerüst" der Gebäude vernachlässigbar sind und daß die dafür aufgewendete Energie gegenüber der Heizenergie klein ist.

(5) "Neue Wohngebäude": Dieser Prozeß umfaßt die nach der Zeit $t_{0}$ neu erstellten Wohngebäude. Analog zu den Prozessen (3) und (4) werden sie mit Energie beheizt $\left(T_{15}(t)\right)$, neu wird hier der aus der "Herstellung" kommende Beton $\left(A_{25}(t)\right)$ gelagert. Unter der Annahme, daß innert der betrachteten Zeitspanne von 50 Jahren keine neu erstellten Wohngebäude abgebrochen werden und an deren "Betongerüst" keine ins Gewicht fallenden Änderungen vorgenommen werden, verläßt diesen Prozeß keine Materie.

(6) "Abbruch": Hierdurch werden alte und sanierte Wohngebäude abgebrochen und das dabei gewonnene Material aufbereitet und weiterverteilt. Ein Teil des Betons aus den sanierten Gebäuden $\left(A_{46}(t)\right)$ und den alten Wohngebäuden $\left(A_{36}(t)\right)$ findet so als Recyclingkies (das heißt Betongranulat, Fluß $\left.A_{62}(t)\right)$ im Prozeß "Herstellung" eine Wiederverwendung, der Rest $\left(A_{68}(t)\right)$ verläßt als Deponiegut das System. Die für diesen Prozeß benötigte Energie ist gegenüber den anderen Energieflüssen vernachlässigbar klein und wird deshalb nicht berücksichtigt.

\subsection{Mathematische Beschreibung des Metabolismus}

Die mathematische Beschreibung wird nach erprobtem Schema [2] folgendermaßen durchgeführt:

- Aufstellen der Systemvariablen;

- Aufstellen der Bilanzgleichungen;

- Beschreibung des Modellansatzes mit der Einführung von Systemparametern.

Sie läßt sich aufteilen in eine Beschreibung für Materie und Energie.

\subsubsection{Modellbeschreibung für Materie}

\section{Systemvariablen}

Die 12 Systemvariablen für Materie sind die 5 Materiemengen $M^{(2)}(t)$, $M^{(3)}(t), M^{(4)}(t), M^{(5)}(t), M^{(6)}(t)$ in Tonnen pro Einwohner [t/E] und die 7 Materieflüsse $A_{25}(t), A_{34}(t), A_{36}(t), A_{46}(t), A_{62}(t)$, 
$A_{68}(t), A_{72}(t)$ in Tonnen pro Einwohner und $\mathrm{Jahr}[\mathrm{t} / \mathrm{E} \mathrm{a}]$.

\section{Bilanzgleichungen}

Die Ableitungen der Materiemengen nach der Zeit, $\dot{M}^{(\mathrm{i})}(t)$, sind nach dem Massenerhaltungssatz die Differenz zwischen den zugeführten Gütern und den abgeführten Gütern eines Prozesses. Für die 5 Prozesse, die durch entsprechende Differentialquotienten beschrieben werden, gelten also die folgenden Bilanzgleichungen:

$\dot{M}^{(2)}(t)=A_{62}(t)+A_{72}(t)-A_{25}(t)$
$\dot{M}^{(3)}(t)=-A_{34}(t)-A_{36}(t)$
$\dot{M}^{(4)}(t)=A_{34}(t)-A_{46}(t)$
$\dot{M}^{(5)}(t)=A_{25}(t)$
$\dot{M}^{(6)}(t)=A_{36}(t)+A_{46}(t)-A_{62}(t)-A_{68}(t)$

\section{Modellansätze}

Die Grundidee dieses Modells beruht auf der Annahme, daß Änderungen am Baubestand wie Neubau, Abbau und Sanierungen durch gesellschaftliche Faktoren (ökonomische, demographische, politische) entschieden werden. Das Bauwerk wird deshalb stärker von wechselnden gesellschaftlichen Rahmenbedingungen beeinflußt als durch den eigenen physiologischen Zustand. Beispielsweise entscheidet über den Abbruch eines Gebäudes viel mehr die Rentabilität, Eigentumsverhältnisse, Änderungen der unmittelbaren Umgebung als etwa das Alter des Gebäudes. Aus diesem Grund werden hier die Flüsse in und aus den Gebäuden durch Parameterfunktionen vorgegeben $\left(P_{\mathrm{i}}(t)\right.$ in den
Gleichungen (6a)-(9a)). Sie könnten beispielsweise durch Modelle aus sozialwissenschaftlichen Disziplinen bestimmt oder, wie es hier im weiteren durchgespielt werden soll, im Sinne von Szenariovorgaben gesetzt werden.

Zudem wird angenommen, daß in den Prozessen "Abbruch Wohngebäude" und "Herstellung Wohngebäude" zu keiner Zeit eine Lagerbildung stattfindet, Gleichungen (10a) und (11a), und daß der Recyclingkies-Fluß proportional zur Abbruch-Menge ist, Gleichung (12a).

\begin{tabular}{|lr|}
$A_{25}(t)=P_{1}(t)$ & $(6 \mathrm{a})$ \\
$A_{34}(t)=P_{2}(t)$ & $(7 \mathrm{a})$ \\
$A_{36}(t)=P_{3}(t)$ & $(8 \mathrm{a})$ \\
$A_{46}(t)=P_{4}(t)$ & $(9 \mathrm{a})$ \\
$M^{(2)}(t)=0$ & $(10 \mathrm{a})$ \\
$M^{66}(t)=0$ & $(12 \mathrm{a})$ \\
$A_{62}(t)=r(t)\left[A_{36}(t)+A_{46}(t)\right]$ & \\
$P_{1}(t), P_{2}(t), P_{3}(t), P_{4}(t):$ & \\
$\quad$ Parameterfunktionen der Betonflüsse \\
in und aus Wohngebäuden (in t/E a) \\
$r(t):$ Parameterfunktion der Recyclingrate \\
\multicolumn{2}{l}{ (dimensionslos oder in Prozent) }
\end{tabular}

\subsubsection{Modellbeschreibung für Energie}

\section{Systemvariablen}

Die 14 Systemvariablen für Energie sind die 5 Energiemengen $E^{(1)}(t), E^{(2)}(t)$, $E^{(3)}(t), E^{(4)}(t), E^{(5)}(t)$ in Megajoule pro Einwohner $[\mathrm{MJ} / \mathrm{E}]$ und die 9 Energieflüsse $T_{12}(t), T_{13}(t), T_{14}(t), T_{15}(t), T_{91}(t)$, $T_{210}(t), T_{310}(t), T_{410}(t), T_{510}(t)$ in Megajoule pro Einwohner und Jahr [MJ/E a].

Tabelle 1. Annahmen für die in durchschnittlichen neuen Gebäuden verwendeten Materialien und die bei Herstellung und Transport aufgewendete Energie.

\begin{tabular}{|c|c|c|c|}
\hline Get & $\begin{array}{l}\text { aterial pro } \\
\text { qudevolumen } \\
{\left[\mathrm{kg} / \mathrm{m}^{3}\right]}\end{array}$ & $\begin{array}{l}\text { Spezifische Energie } \\
\text { für } \\
\text { Materialherstellung } \\
{[\mathrm{MJ} / \mathrm{kg}]^{\text {al }}}\end{array}$ & $\begin{array}{l}\text { Spezifische Energie } \\
\text { für die Herstellung } \\
\text { der Gebaude } \\
{\left[\mathrm{MJ} / \mathrm{m}^{3}\right]^{\circ)}}\end{array}$ \\
\hline $\begin{array}{l}\text { Beton } \\
\quad \text { davon Armierung c) } \\
\text { davon Zement }\end{array}$ & $\begin{array}{r}500 \\
13 \\
75\end{array}$ & $\begin{array}{c}0.8 \\
10 \\
\\
3.7\end{array}$ & $\begin{array}{r}410 \\
130 \\
280\end{array}$ \\
\hline Glas & 3 & 8 & 25 \\
\hline Holz d) & 10 & 3 & 30 \\
\hline Aluminiumc) & 1 & 70 & 70 \\
\hline Kunststoff & 3 & 4 & 10 \\
\hline Stahlo) & 3 & 10 & 30 \\
\hline $\begin{array}{l}\text { Total (Energie Material) } \\
\left.\text { Energie Transport }{ }^{\Theta}\right) \\
\end{array}$ & 520 & 1.1 & \\
\hline \multicolumn{4}{|l|}{ Total Energie Herstellung } \\
\hline
\end{tabular}

\section{Bilanzgleichungen}

Analog zu den Gleichungen (1a)-(5a) für Materie lassen sich die Bilanzgleichungen für Energie aufstellen. $\dot{E}^{(i)}(t)$ sind hier die zeitlichen Ableitungen der Energiemengen:

$$
\begin{aligned}
\dot{E}^{(1)}(t)= & T_{91}(t)-T_{12}(t)-T_{13}(t) \\
& -T_{14}(t)-T_{15}(t) \\
\dot{E}^{(2)}(t)= & T_{12}(t)-T_{210}(t) \\
\dot{E}^{(3)}(t)= & T_{13}(t)-T_{310}(t) \\
\dot{E}^{(4)}(t)= & T_{14}(t)-T_{410}(t) \\
\dot{E}^{(5)}(t)= & T_{15}(t)-T_{510}(t)
\end{aligned}
$$

\section{Modellansätze}

Für alle Prozesse mit Energieflüssen ist anzunehmen, daß die Energielager klein gehalten werden und sich über den Zeithorizont eines Jahres nicht wesentlich ändern. Sie werden darum vereinfachend auf null gesetzt, Gleichungen (6b)-(10b). Ihre zeitliche Ableitung ist somit auch null.

Die Energie für die Herstellung der Gebäude wird als proportional zum Betonfluß angenommen, da dieser sowohl den Material- als auch den Energieaufwand bei der Herstellung dominiert (Gleichung (11b); vergleiche auch Tabelle 1).

$$
\begin{array}{|lr}
E^{(1)}(t)=E^{(2)}(t)=E^{(3)}(t)=E^{(4)}(t)=E^{(5)}(t)=0 \\
& (6 \mathrm{~b})-(10 \mathrm{~b}) \\
T_{12}(t)=e_{2}(t) A_{25}(t) & (11 \mathrm{~b}) \\
T_{13}(t)=e_{3}(t) V_{3}(t) & (12 \mathrm{~b}) \\
T_{14}(t)=e_{4}(t) V_{4}(t) & (13 \mathrm{~b}) \\
T_{15}(t)=e_{5}(t) V_{5}(t) & (14 \mathrm{~b}) \\
e_{2}(t): & \text { Parameterfunktion des spezifischen } \\
& \text { Energieverbrauchs für die Herstellung } \\
& \text { von Wohngebäuden (in MJ/t) } \\
e_{3}(t), & e_{4}(t), e_{5}(t): \\
& \text { Parameterfunktionen des spezifischen } \\
& \text { Energieverbrauchs für das Heizen der } \\
\text { alten, sanierten und neuen Wohn- } & \\
\text { gebäude (in MJ/m }{ }^{3} \text { a) } & \\
V_{3}(t), & V_{4}(t), V_{5}(t): \\
\text { Parameterfunktionen der Volumen- } & \\
\text { entwicklung von alten, sanierten und } & \text { neuen Wohngebäuden (in m } \left.{ }^{3} / E\right)
\end{array}
$$

Für die in den Gebäuden verbrauchte Heizenergie wird angenommen, daß sie einerseits abhängt von der Größe der Gebäude - dem Gebäudevolumen - und anderseits von einer gebäudespezifischen Energiekennzahl, die im wesentlichen den Einfluß der Konstruktion eines Gebäudes (Materialien, Geometrie, Exposition, Technik), aber auch des Klimas, 
des Benutzerverhaltens, der Nutzung und so weiter angibt (Gleichungen (12b)(14b)). Der Vorteil dieses Ansatzes liegt darin, daß der Einfluß auf den Energieverbrauch durch Änderungen von Gebäudevolumen und Haustechnik separat untersucht werden kann. Die Aufteilung in alte, sanierte und neue Gebäude berücksichtigt die grundsätzlich unterschiedlichen Entwicklungen der Energiekennzahlen für diese drei Gebäudetypen.

\subsubsection{Die Lösungen der Systemgleichungen}

Mit den 12 Gleichungen für Materie und 14 Gleichungen für Energie lassen sich nun für alle Systemvariablen die allgemeinen Lösungen berechnen.

\section{Lösungen für Materie.}

$A_{25}(t)=P_{1}(t)$
$A_{34}(t)=P_{2}(t)$
$A_{36}(t)=P_{3}(t)$
$A_{46}(t)=P_{4}(t)$
$A_{62}(t)=r(t)\left[P_{3}(t)+P_{4}(t)\right]$
$A_{68}(t)=[1-r(t)]\left[P_{3}(t)+P_{4}(t)\right]$
$A_{72}(t)=P_{1}(t)-r(t)\left[P_{3}(t)+P_{4}(t)\right]$
$M^{(2)}(t)=0$
$M^{(3)}(t)=\int_{t_{0}}^{t}\left[-P_{2}\left(t^{\prime}\right)-P_{3}\left(t^{\prime}\right)\right] \mathrm{d} t^{\prime}+M^{(3)}\left(t_{0}\right)$
$M^{(4)}(t)=\int_{t_{0}}^{t}\left[P_{2}\left(t^{\prime}\right)-P_{4}\left(t^{\prime}\right)\right] d t^{\prime}+M^{(4)}\left(t_{0}\right)$
$M^{(5)}(t)=\int_{t_{0}}^{t} P_{1}\left(t^{\prime}\right) d t^{2}+M^{(5)}\left(t_{0}\right)$
$M^{(6)}(t)=0$

\section{Lösungen für Energie.}

$$
\begin{aligned}
T_{12}(t) & =T_{210}(t)=e_{2}(t) P_{1}(t) \\
T_{13}(t) & =T_{310}(t)=e_{3}(t) V_{3}(t) \\
T_{14}(t) & =T_{410}(t)=e_{4}(t) V_{4}(t) \\
T_{15}(t) & =T_{510}(t)=e_{5}(t) V_{5}(t) \\
T_{91}(t) & =e_{2}(t) P_{1}(t)+e_{3}(t) V_{3}(t)+e_{4}(t) V_{4}(t) \\
& +e_{5}(t) V_{5}(t) \\
E^{(1)}(t) & =E^{(2)}(t)=E^{(3)}(t)=E^{(4)}(t)=E^{(5)}(t)=0
\end{aligned}
$$

Die Parameterfunktionen $P_{1}(t), P_{2}(t)$, $P_{3}(t), P_{4}(t), r(t), e_{2}(t), e_{3}(t), e_{4}(t), e_{5}(t)$, $V_{3}(t), V_{4}(t), V_{5}(t)$ und die Anfangswerte für die Materielager in den drei Gebäudeklassen $M^{(3)}\left(t_{0}\right), M^{(4)}\left(t_{0}\right), M^{(5)}\left(t_{0}\right)$ sind zu bestimmen. Wie schon vorher angedeutet, sollen und können hier für die Parameterfunktionen keine dynamischen
Modellbeziehungen eingesetzt werden. Vielmehr soll für den allgemeingültig formulierten dynamischen Modellansatz ein Spezialfall - der vereinfachte, quasistationäre Fall - diskutiert werden. Dabei werden für die Parameterfunktionen einfache lineare Beziehungen von Parametern eingesetzt. Die Lösungen für die Systemvariablen bleiben auf diese Weise einfach analytisch zugänglich und unmittelbar nachvollziehbar. Durch Variation der Parameter können nun verschiedene Szenarios durchgespielt werden. Anhand von drei Szenarios sei erläutert, wie das Modell benutzt werden kann.

Für die Parameterfunktionen werden folgende Parameterbeziehungen eingesetzt:

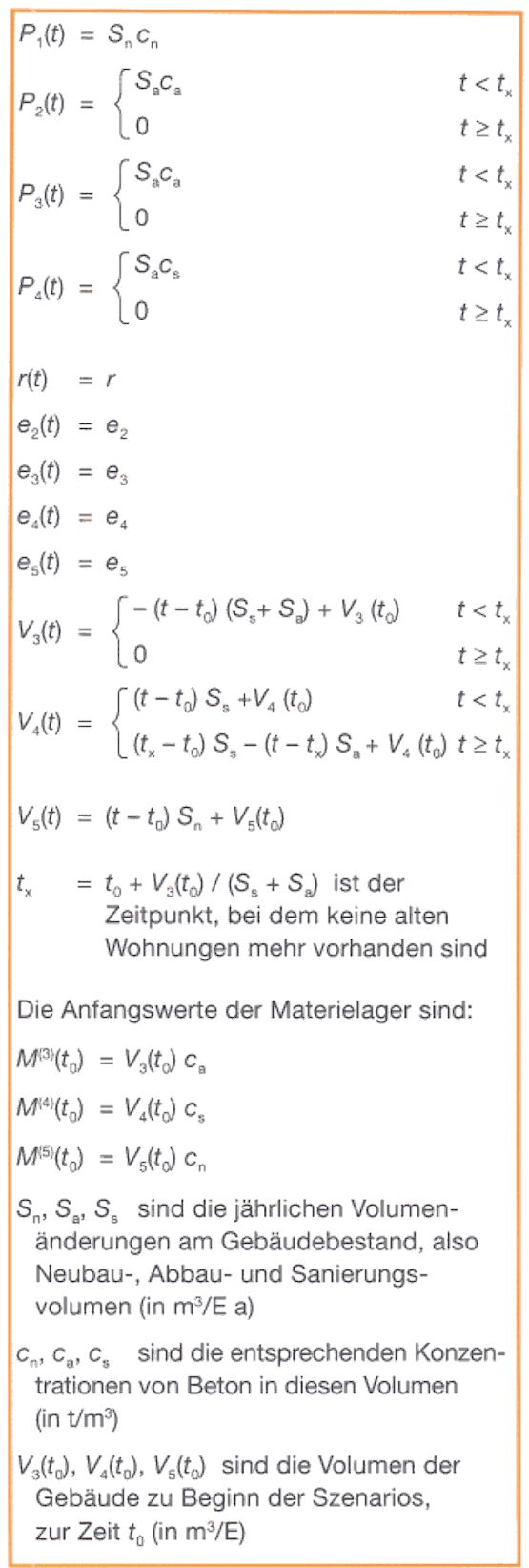

\subsection{Drei Szenarios zur Entwicklung der Wohngebäude}

Es sollen hier zwei grundsätzlich verschiedene "ökologische" Szenarios und ein Referenzszenario untersucht werden. Aus Gründen der Datenverfügbarkeit wird als Region die gesamte Schweiz betrachtet. Da sich rund vier Fünftel des Gebäudeparks in dicht besiedelten Gebieten wie dem Mittelland und der Nordwestschweiz befinden, wird die gesamte Schweiz in erster Näherung als repräsentativ für dicht besiedelte Regionen angesehen. Für alle Szenarios wird als Ausgangspunkt das Jahr 2000, also $t_{0}=2000$, gesetzt und der Zeithorizont auf die nächsten 50 Jahre begrenzt (wie schon erwähnt, wird für neue Gebäude eine Lebensdauer von mindestens 50 Jahren vorausgesetzt). Die Szenarios werden für eine konstante Bevölkerungszahl von 7.4 Millionen Einwohner gerechnet. Sie lassen sich fogendermaßen charakterisieren:

- Das Szenario "Business as usual" (B.a.u.) veranschaulicht, was geschähe, wenn die heute geübte Praxis beibehalten wird. Das heutige Wachstum des Wohngebäudevolumens wird fortgesetzt: Das Neubauvolumen bleibt unverändert gro $B$ und das Abbruchvolumen sehr klein. Neubauten und Sanierungen werden nach den heute üblichen Standards ausgeführt.

- Im Szenario "Erhalten" wird die Strategie der Minimierung von Sandund Kiesverbrauch verfolgt, indem das Neubauvolumen auf ein substanzerhaltendes Minimum zurückgeht: Es reduziert sich auf das Äquivalent des heutigen Abbruchvolumens, so daß insgesamt kein Wachstum des Gebäudevolumens mehr stattfindet.

- Beim Szenario "Umbauen" wird zur Minimierung des Energieverbrauchs das gesamte Wohngebäudevolumen innert 50 Jahren durch neue Niedrigenergiegebäude ersetzt. Dafür ist es notwendig, das Abbruchvolumen massiv zu steigern. Aber auch hier soll das Gebäudevolumen insgesamt nicht zunehmen das heißt, das Neubauvolumen muß mit dem Abbruchvolumen Schritt halten.

Die beiden Szenarios "Erhalten". und "Umbauen" unterscheiden sich nur in Abbruch- und Neubauvolumen, alle anderen Parameter sind so gewählt, daß die heutigen technischen Möglichkeiten zur Minimierung von Beton- und Energieverbrauch ausgeschöpft werden. Tabelle 2 gibt einen Überblick über die gewählten Parameter für die drei Szenarios der quasistationären Betrachtung. 
Tabelle 2. Drei Szenarios zur Entwicklung der Wohngebäude und die hierfür gewählten Parameter, welche die Parameterfunktionen des quasistationären Falls bestimmen. In geschweiften Klammern sind die numerierten Verweise auf die Erläuterungen zu diesen Zahlen im Text angegeben.

\begin{tabular}{|c|c|c|c|c|c|}
\hline \multirow[t]{2}{*}{ Parameter } & \multirow[t]{2}{*}{ Symbol } & \multirow[t]{2}{*}{ Einheit } & \multicolumn{3}{|c|}{ Werte für Szenario: } \\
\hline & & & "B.a.u." & "Erhalten" & "Umbau" \\
\hline Volumen der alten Wohngebäude zu Beginn & $V^{(3)}\left(t_{0}\right)$ & $\mathrm{m}^{3} / \mathrm{E}$ & & 210 & \\
\hline Volumen der sanierten Wohngebäude zu Beginn & $V^{(4)}\left(t_{0}\right)$ & $\mathrm{m}^{3} / \mathrm{E}$ & & $\{2\}$ & \\
\hline Volumen der neuen Wohngebäude zu Beginn & $V^{(5)}\left(t_{0}\right)$ & $\mathrm{m}^{3 / E}$ & & $\{2\}$ & \\
\hline Abbruchvolumen & $S_{a}$ & $\mathrm{~m}^{3} / \mathrm{E} a$ & $0.1\{3\}$ & $0.1\{4\}$ & $4.2\{5\}$ \\
\hline Sanierungsvolumen & $s_{s}$ & $\mathrm{~m}^{3} / \mathrm{E} a$ & $7 \quad\{6\}$ & 20 & $\{7\}$ \\
\hline Neubauvolumen & $S_{n}$ & $\mathrm{~m}^{3} / \mathrm{E} a$ & $2.8\{8\}$ & $0.1\{5\}$ & $4.2\{9\}$ \\
\hline Beton-Konzentration alter Wohngebäude & $c_{\mathrm{a}}$ & $t / m^{3}$ & & $0.38\{10\}$ & \\
\hline Beton-Konzentration sanierter Wohngebäude & $c_{\mathrm{s}}$ & $t / m^{3}$ & & $0.38\{11\}$ & \\
\hline Beton-Konzentration neuer Wohngebäude & $c_{n}$ & $\mathrm{t} / \mathrm{m}^{3}$ & & $0.5\{12\}$ & \\
\hline Spezifischer Energieverbrauch für die Herstellung & $e_{2}$ & $\mathrm{MJ} / \mathrm{t}$ & & $1320\{13\}$ & \\
\hline Spezifischer Heizenergieverbrauch alter Wohngebäude & $e_{3}$ & $\mathrm{MJ} / \mathrm{m}^{3} \mathrm{a}$ & & $\{14\}$ & \\
\hline Spezifischer Heizenergieverbrauch sanierter Wohngebäude & $e_{4}$ & $\mathrm{MJ} / \mathrm{m}^{3} \mathrm{a}$ & $\{15\}$ & 60 & $\{16\}$ \\
\hline Spezifischer Heizenergieverbrauch neuer Wohngebäude & $e_{5}$ & $\mathrm{MJ} / \mathrm{m}^{3} \mathrm{a}$ & $\{17\}$ & 10 & $\{18\}$ \\
\hline Recyclingrate Beton & $r$ & 4 & $0.33\{19\}$ & 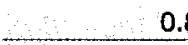 & $\{20\}$ \\
\hline Zeitdauer bis keine alten Wohngebäude mehr vorhanden & $t_{x}-t_{0}$ & a & 29.6 & 10.4 & 8.7 \\
\hline
\end{tabular}

\section{Grundlagen für die Wahl der}

Parameter

\{1 $\}$ Da $t_{0}=2000$, sind die "alten Wohngebäude" der Bestand an Wohngebäuden im Jahr 2000. Aus der amtlichen Statistik ${ }^{[6]}$ sind die Anzahl Wohnungen und die durchschnittliche Wohnfläche pro Wohnung für $1990 \mathrm{zu}$ entnehmen. Diese Fläche kann nach bekanntem Verfahren ${ }^{[8]}$ mit dem Faktor 1.3 in Bruttogeschoßfläche (BGF) umgerechnet werden und mit dem Faktor 1.43 weiter in die Bruttonutzfläche (BNF). Durch Multiplikation mit der mittleren Geschoßhöhe $(2.6 \mathrm{~m})$ ergibt sich daraus das umbaute Volumen der Wohngebäude für 1990 von 1370 Millionen Kubikmeter. Für das Jahr 2000 werden 1560 Millionen Kubikmeter geschätzt (210 Kubikmeter pro Einwohner).
\{2\} Einfachheitshalber werden Volumen (und damit auch Betoninhalt) der bis zum Jahr 2000 sanierten und neugebauten Wohngebäude auf null gesetzt. Real bis zu diesem Zeitpunkt schon sanierte Gebäude sind in der bezüglich spezifischen Energieverbrauchs sowieso breitgefächerten Gruppe alter Wohngebäude berücksichtigt. Es wird angenommen, daß sie ein zweites Mal im Sinne der Szenariovorgaben sanierbar sind.

\{3\} Abbruchvolumen der Jahre 19851994 im Mittel: 0.7 Mio. $\mathrm{m}^{3} / \mathrm{a}$; berechnet aus der Anzahl abgebrochener Wohnungen ${ }^{\text {[9] }}$ und dem mittleren Volumen.

\{4\} Annahme: Abbruchvolumen so gering wie heute.

\{5\} Das Abbruchvolumen wird gleich dem Neubauvolumen gesetzt, um das Gebäudevolumen konstant zu halten

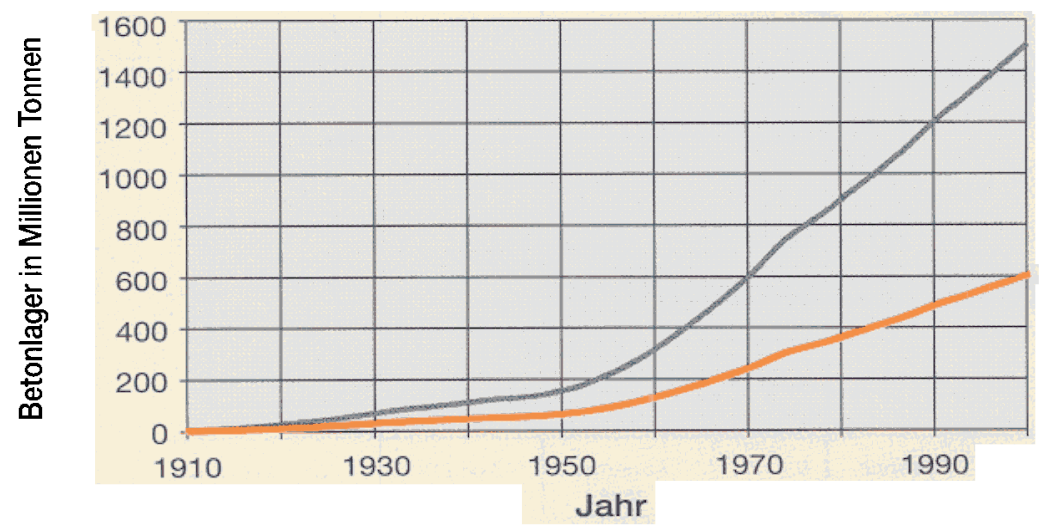

Betonlager total

Betonlager in Wohngebäuden

Figur 2. Durch Aufsummieren der jährlichen Betonproduktion läßt sich der in den Bauten der Schweiz gelagerte Beton abschätzen (graue Linie). Rund $40 \%$ davon befindet sich in Wohngebäuden (gelbe Linie).

(siehe auch Beschreibung der Szenarios).

\{6\} Aufgrund der in den Jahren 1986 und 1990 renovierten Wohnungen [6] und der mittleren Wohnfläche pro Wohnung wurde der jährliche Volumenanteil auf 50 Mio. $\mathrm{m}^{3}$ geschätzt. Es wird in erster Näherung angenommen, daß eine Renovation (nach [6]: $» .$. jede Veränderung oder Umgestaltung, die eine merkliche Wertsteigerung des betreffenden Gebäudes zur Folge hat«) immer auch eine energetische Sanierung umfaßt. Für das Jahr 2000 wird mit einem Renovationsvolumen von 52 Mio. $\mathrm{m}^{3} / \mathrm{a}$ gerechnet.

\{7\} Annahme: Einführung eines Gesetzes, daß innert 10 Jahren alle Wohngebäude energetisch saniert werden müssen.

\{8\} Neubauvolumen der Jahre 19851994 im Mittel: 21 Mio. $\mathrm{m}^{3} / \mathrm{a}$; berechnet aus der Anzahl neugebauter Wohnungen ${ }^{[9]}$ und dem mittleren Volumen. Zuwachs durch Umbau wird vernachlässigt.

\{9\} Das - über 10 Jahre gemittelt bisher höchste Neubauvolumen pro Einwohner (Ende 60er bis Anfang 70er Jahre) betrug rund $4.4 \mathrm{~m}^{3} / \mathrm{E}$ a. Es wird daher angenommen, daß über längere Zeit ein Neubauvolumen von über 4.2 $\mathrm{m}^{3} / \mathrm{E}$ a (das entspricht 2 Prozent des heutigen Bestandes) volkswirtschaftlich nicht durchzuhalten ist.

$\{10\}$ Aufgrund des Ausstoßes der heimischen Zementindustrie von 1910 bis zum Jahr $2000{ }^{[10]}$ (1995-2000: Schätzung) kann - unter der Annahme, daß sämtlicher Zement zu Beton verarbeitet wurde - die Produktion von Beton abgeschätzt werden. Die Summe dieser jährlichen Verbräuche entspricht der 
total verbauten Menge Beton. Nach Angaben der Hersteller [ ${ }^{[0]}$ entfallen auf den Zementverbrauch des privaten Wohnungsbaus etwa 40 Prozent. Für den Kanton Solothurn wurde der Anteil auf 50 Prozent geschätzt ${ }^{[11]}$. Wir rechnen hier mit einem Anteil von 40 Prozent (siehe Figur 2). Der an Wohnbauten bereits abgebrochene Beton wird mit den abgebrochenen Wohnungen [9] auf 2 Prozent geschätzt. Das in Wohngebäuden aufgebaute Lager $M^{(3)}\left(t_{0}\right)$ beträgt demnach etwa 600 Millionen Tonnen. Mit dem bekannten Volumen ergibt sich $c_{\mathrm{a}}=M^{(3)}\left(t_{0}\right) / V^{(3)}\left(t_{0}\right)=0.38$ Tonnen Beton pro Kubikmeter Gebäuderaum.

\{11\} Annahme: Durch eine energetische Sanierung wird die Betonkonzentration nicht verändert.

$\{12\}$ Ein fiktives Modellhaus mit durchschnittlicher Geometrie eines Wohngebäudes (Boden, Decken und Untergeschoßwände aus Beton, Außen- und Innenwände zu $70 \%$ aus Beton, $20 \mathrm{~cm}$ Wandstärke) hat eine Betonkonzentration von $0.5 \mathrm{t} / \mathrm{m}^{3}$. Ein Zürcher Indexhaus ${ }^{[2]} \mathrm{kommt}$ auf $0.4 \mathrm{t} / \mathrm{m}^{3}$. Niedrigenergiehäuser, die für die Speicherung der passiven Sonnenenergie eine gewisse Masse brauchen, weisen ebenfalls einen Wert um $0.4 \mathrm{t} / \mathrm{m}^{3}$ auf ${ }^{[12]}$. Nimmt man analog zur Berechnung von $M^{(3)}\left(t_{0}\right)$ an, daß 40 Prozent des verbrauchten Betons auf den Wohnungsbau entfallen, resultiert für die Zeitspanne 1986-1990 eine Betonkonzentration von $0.55 \mathrm{t} / \mathrm{m}^{3}$. Wir rechnen mit $c_{\mathrm{n}}=0.5 \mathrm{t} / \mathrm{m}^{3}$.

\{13\} Gemäß den Annahmen in Tabelle 1 werden pro Kubikmeter Gebäuderaum rund 660 Megajoule Energie bei der Herstellung und dem Transport verbraucht. Bezogen auf die 0.5 Tonnen Beton pro Kubikmeter entspricht dies $1.32 \mathrm{MJ} / \mathrm{kg}$ oder $1320 \mathrm{MJ} / \mathrm{t}$ Beton.

\{14\} Entspricht der Energiekennzahl für Wärme $E_{\mathrm{w}}=520 \mathrm{MJ} / \mathrm{m}^{2}$ a. Nach Wick ${ }^{[14]}$ betrug $E_{\mathrm{w}}(1989)=580 \mathrm{MJ} / \mathrm{m}^{2}$ a für Mehrfamilienhäuser. Mit der Annahme Energiebezugsfläche (EBF) $=$ BGF und dem Wärmeverbrauch der Haushalte ${ }^{[15]}$ berechnet $\operatorname{man} E_{\mathrm{w}}(1990)=570 \mathrm{MJ} / \mathrm{m}^{2}$ a. $\{15\}$ Entspricht $E_{\mathrm{w}}=375 \mathrm{MJ} / \mathrm{m}^{2}$ a für gute Sanierungen ${ }^{[14]}$. Zum Vergleich: Sollwert des SIA (Schweizerischer Ingenieur- und Architekten-Verein) $E_{\mathrm{w}}=$ $500 \mathrm{MJ} / \mathrm{m}^{2}$ a.

$\{16\}$ Entspricht $E_{\mathrm{w}}=230 \mathrm{MJ} / \mathrm{m}^{2}$ a für mögliche Sanierungen ${ }^{[16]}$. Zum Vergleich: Sollwert (SIA) $E_{\mathrm{w}}=500 \mathrm{MJ} / \mathrm{m}^{2}$ a. $\{17\}$ Entspricht $E_{\mathrm{w}} \approx 250 \mathrm{MJ} / \mathrm{m}^{2}$ a für gute Neubauten ${ }^{[14]}$.

$\{18\}$ Aufgrund einiger Werte ausge- wählter Niedrigenergiegebäude wird die Energiekennzahl $E_{\mathrm{w}}=40 \mathrm{MJ} / \mathrm{m}^{2}$ a angenommen (Siedlung Boller [17]: $E_{\mathrm{w}}=$ $8 \mathrm{MJ} / \mathrm{m}^{2}$ a; Niedrigenergie-Mehrfamilienhaus BL [18]: $E_{\mathrm{w}}=54 \mathrm{MJ} / \mathrm{m}^{2} \mathrm{a}$; Trin ${ }^{[12]}$ : $E_{\mathrm{w}}=0 \mathrm{MJ} / \mathrm{m}^{2}$ a; Niederholzboden [12]: $\left.E_{\mathrm{w}}{ }^{\mathrm{w}}=80 \mathrm{MJ} / \mathrm{m}^{2} \mathrm{a}\right)$.

\{19\} Annahme ohne weitere Grundlage. $\{20\}$ Nach dem Brechen von Beton sollte die Feinfraktion (20\% der Masse) verworfen werden, um für den Recyclingbeton die Festigkeit des ursprünglichen Betons zu garantieren ${ }^{[19]}$.

\section{Resultate: \\ Der Metabolismus der Wohngebäude wechselt je nach Szenario erheblich}

In die allgemeinen Lösungen der Systemgleichungen (Abschnitt 3.2.3) wurden für die drei hier ins Auge gefaßten Szenarios die Parameter aus Tabelle 2 eingesetzt. Figur 4 stellt die mit $t=2050$ berechneten Energieund Stoffflüsse für alle drei Fälle dar, Figur 5 die zeitliche Entwicklung des Gebäudevolumens und des Energieverbrauchs. Als Vergleich dient die Aus-

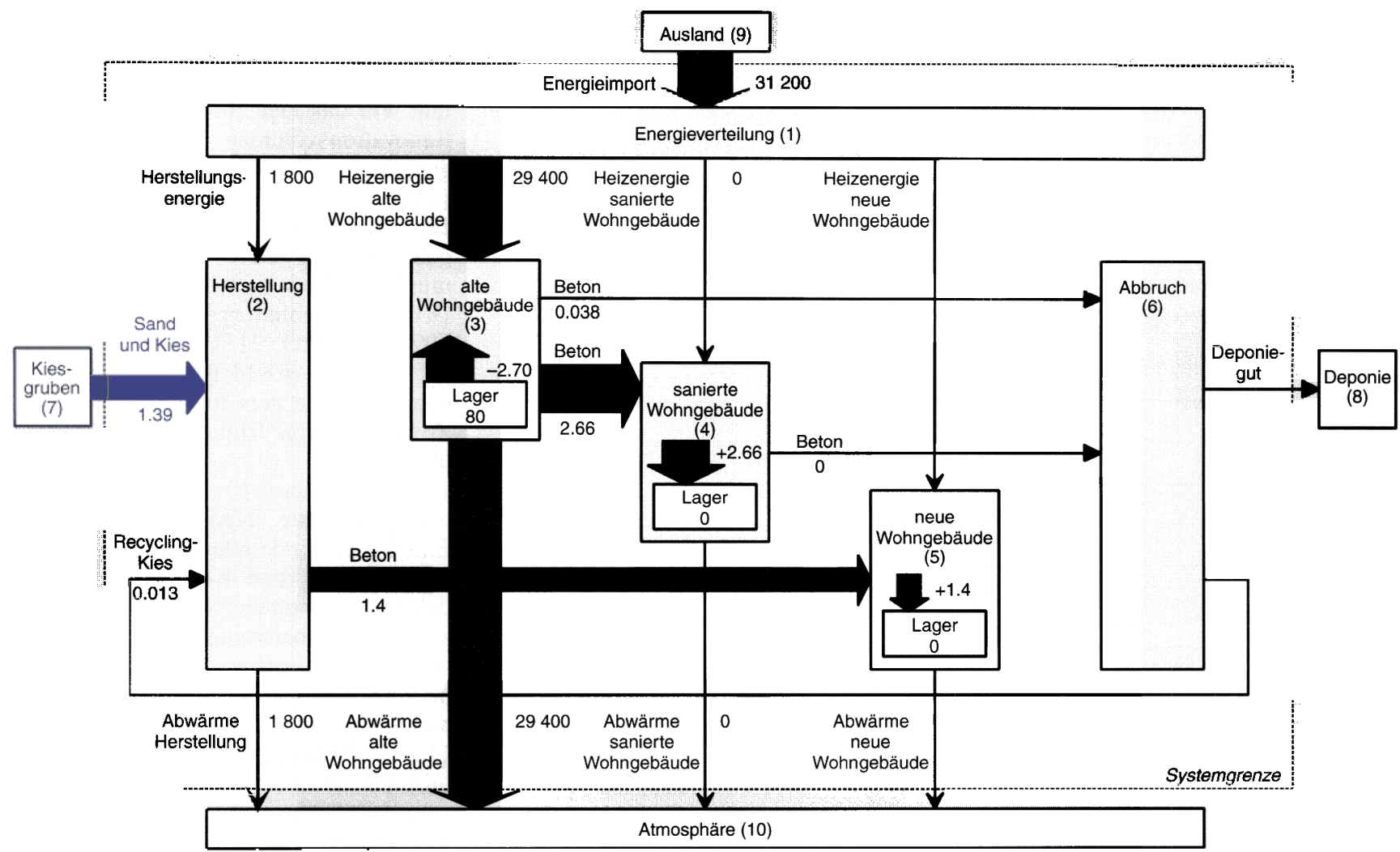

Figur 3. Die Ausgangslage: Materie- und Energieflüsse heute. Sie entsprechen denen des Szenarios "Business as usual" zur Zeit $t=2000$. Während das Lager der neuen Wohngebäude relativ stark wächst, werden kaum alte Gebäude abgebrochen. Materieflüsse sind in Tonnen pro Einwohner und Jahr ( $t / E$ a), Materielager in Tonnen pro Einwohner ( $t / E$ ) und Energieflüsse in Megajoule pro Einwohner und Jahr (MJ/E a) angegeben. 
gangslage, das Szenario "Business as usual" zur Zeit $t=t_{0}=2000$ (Figur 3).

\subsection{Ausgangslage: \\ Das Gebäudelager wächst nach wie vor}

Für das Beheizen der alten Wohngebäude werden in der Schweiz zur Zeit rund 30 Prozent des Energieverbrauchs aufgewendet. Die für die Herstellung benötigte Energie ist um ein bis zwei Größenordnungen geringer. Die alten Wohngebäude werden relativ zügig, mit 3.5 Prozent pro Jahr, in sanierte Gebäude umgewandelt, jedoch kaum abgebrochen (mit dem heutigen Abbruchvolumen dauerte es 2000 Jahre, bis alle Gebäude abgebrochen wären!). Das Lager der neuen Gebäude nimmt mit $1.4 \mathrm{t} / \mathrm{E}$ a rasch zu (Figur 3). Für den gesamten Wohngebäudepark beträgt die jährliche Zuwachsrate des Betonlagers ungefähr 1.7 Prozent. Das entspricht einer Verdoppelung in 50 Jahren. Der Anteil von Recyclingkies am gesamten Kiesverbrauch ist mit etwa 1 Prozent vernachlässigbar und fiele auch bei vollständigem Recycling nicht ins Gewicht.

\subsection{Szenario "Business as usuaf": Energieeinsparungen werden wieder kompensiert}

Bei Fortsetzung der heutigen Praxis zeigte sich die Situation nach 50 Jahren so: Die alten Wohngebäude wurden vollständig in sanierte Wohngebäude übergeführt, die nur noch 70 Prozent der ursprünglich benötigten Energie verbrauchen. Abgebrochen wurden nur 2 von 80 Tonnen Beton der ursprünglich alten Wohngebäude. Hinzugekommen ist hingegen ein in den neuen Wohngebäuden aufgebautes Betonlager der gleichen Größenordnung (siehe Figur 4: oben). Der Energieaufwand für das Heizen dieser zusätzlichen Gebäude kompensiert die durch Sanierung erreichten Einsparungen (Figur 5: oben): Der Gesamtenergieverbrauch hat sich kaum verändert. Allerdings kann nun mit gleicher Energie nahezu das doppelte Gebäudevolumen in Betrieb gehalten werden $\left(345 \mathrm{~m}^{3} / \mathrm{E}\right.$ gegenüber $210 \mathrm{~m}^{3} / \mathrm{E}$ ). An den Kiesflüssen hat sich gegenüber dem Ausgangszustand nichts geändert.

Figur 4. Die Materie- und Energieflüsse der drei Szenarios, wie sie sich im Jahr 2050 präsentieren. Bei einer Strategie des Erhaltens der Gebäudesubstanz werden vor allem die Kiesreserven geschont, während bei einer Umbaustrategie primär der Energieverbrauch massiv reduziert werden könnte. Materieflüsse sind in Tonnen pro Einwohner und Jahr ( $t / E$ a), Materielager in Tonnen pro Einwohner (t/E) und Energieflüsse in Megajoule pro Einwohner und Jahr (MJ/E a) angegeben.
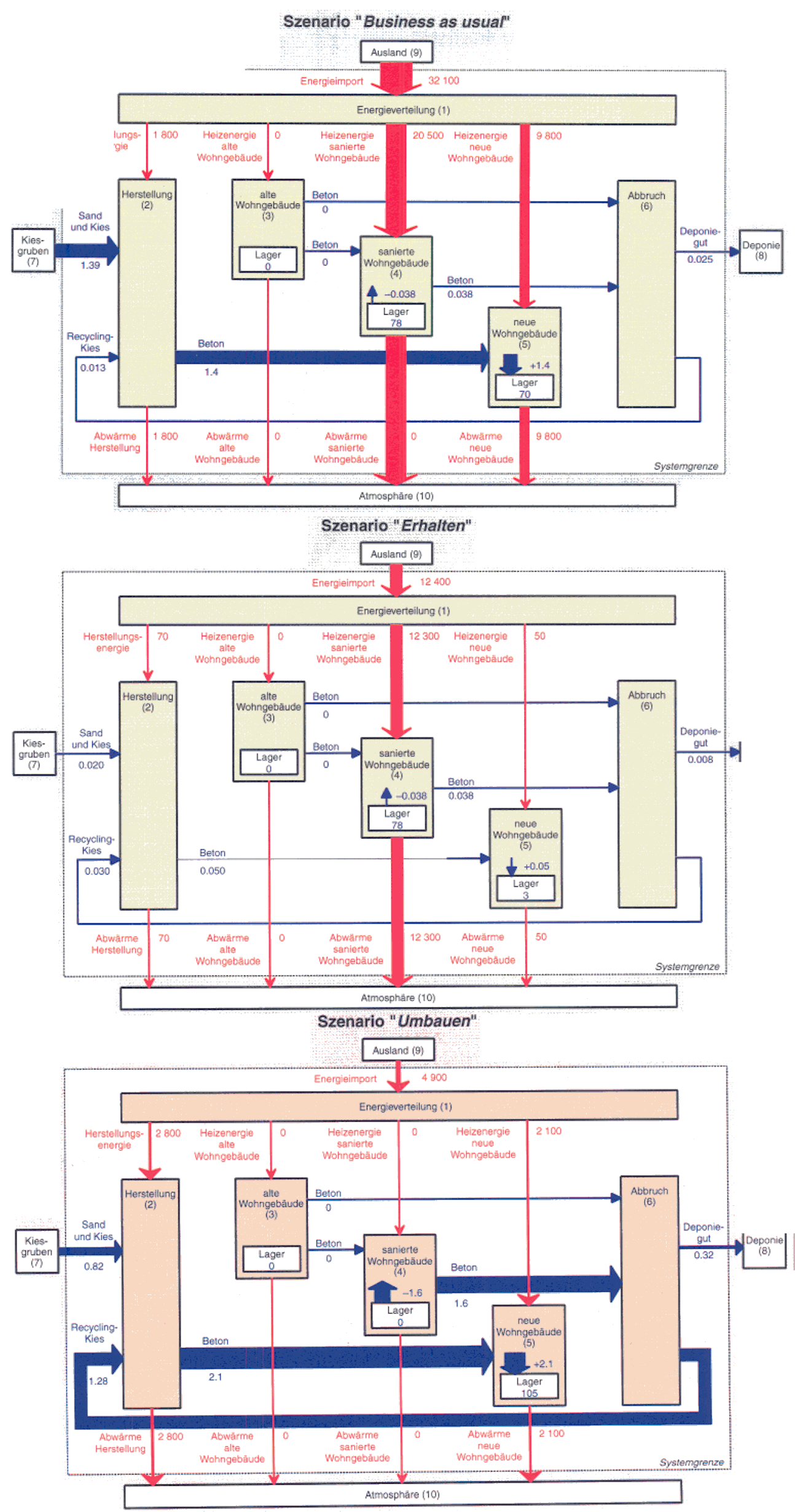


\subsection{Szenario "Erhalten": Der Kiesverbrauch sinkt drastisch}

Die alten Wohngebäude wurden in sanierte Wohngebäude übergeführt, die aufgrund der massiven Sanierung nur noch 40 Prozent der ursprünglichen Energie benötigen. Der geringen Abbruchtätigkeit wegen beträgt das Betonlager der sanierten Gebäude noch $78 \mathrm{t} / \mathrm{E}$, während in den Neubauten nur $3 \mathrm{t} / \mathrm{E}$ hinzugekommen sind. Der Kiesverbrauch konnte um zwei Größenordnungen gesenkt werden (Figur 4: Mitte). Der Gesamtenergieverbrauch wurde um 60 Prozent reduziert, das Einsparpotential bei den bestehenden Gebäuden ist nun aber vollständig ausgeschöpft (Figur 5: Mitte).

\subsection{Szenario "Umbauen": Maximale Energieeffizienz}

Nach 50 Jahren ist der Ersatz der alten und zwischenzeitlich sanierten Wohngebäude durch neue, energieeffizientere Wohngebäude abgeschlossen. Das totale Lager von Beton in den Wohngebäuden ist um rund ein Drittel größer als im Jahr 2000, da die bis dahin gebauten Wohngebäude im Mittel eine geringere Betonkonzentration aufwiesen als die Ersatz-Neubauten (Figur 4: unten). Der Energieverbrauch konnte um beinahe eine Größenordnung gesenkt werden. Die Energie für die Herstellung der Neubauten ist sogar bereits höher als die Betriebsenergie (Figur 5: unten). Der Fluß von Sand und Kies konnte trotz stärkerer Neubautätigkeit

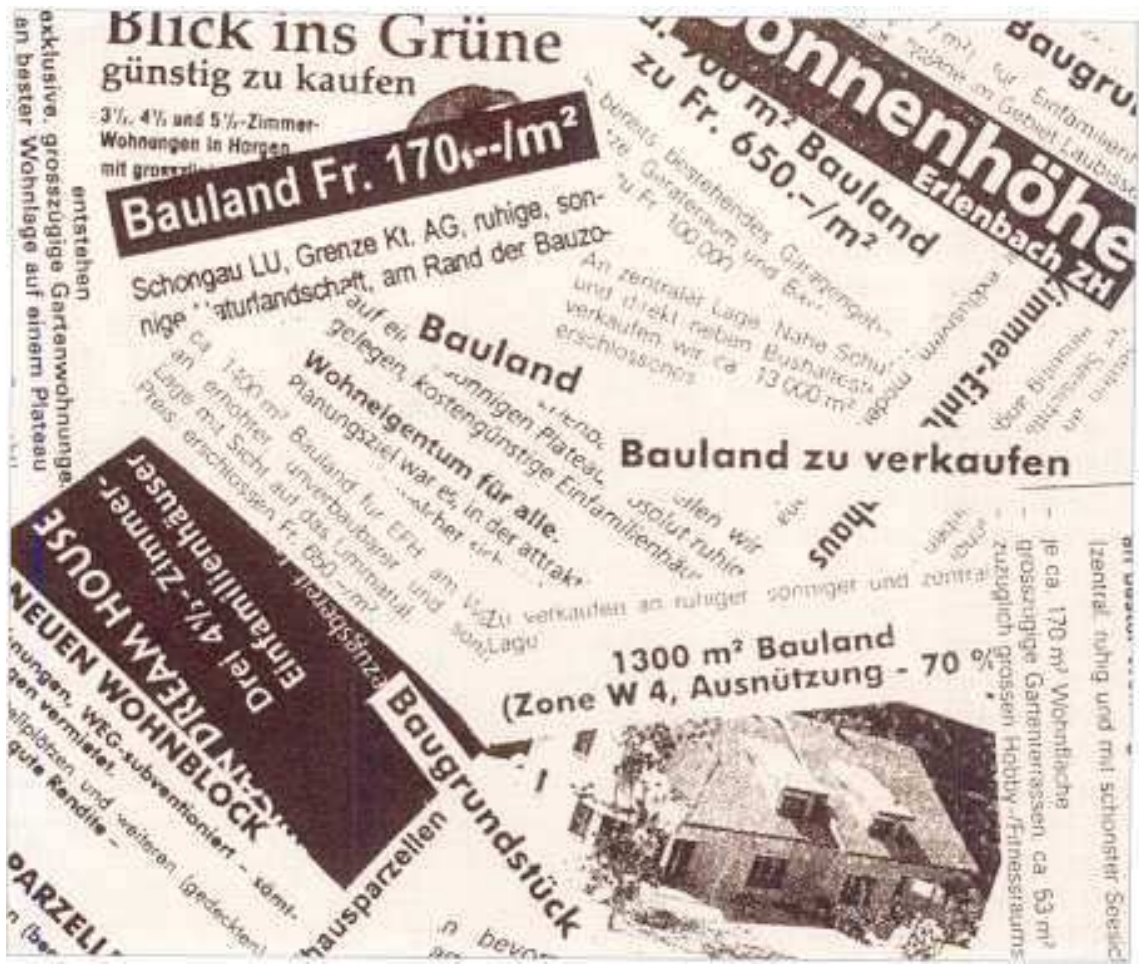

Business as usual: Bauen auf der grünen Wiese ist heute nach wie vor im Trend. Die noch vorhandenen Baulandreserven erlauben ein weiteres Anwachsen des Gebäudeparks.

um 40 Prozent vermindert werden, da wegen der gleichzeitig gesteigerten Abbruchtätigkeit der Beton aus den alten und sanierten Wohngebäuden zur Verfügung stand. Im Gegensatz zu den anderen Szenarios hat hier die hohe Recyclingrate entscheidenden Einfluß auf die Schonung der geogenen Sand- und Kiesressourcen. Mit rund $5000 \mathrm{MJ} / \mathrm{E}$ a

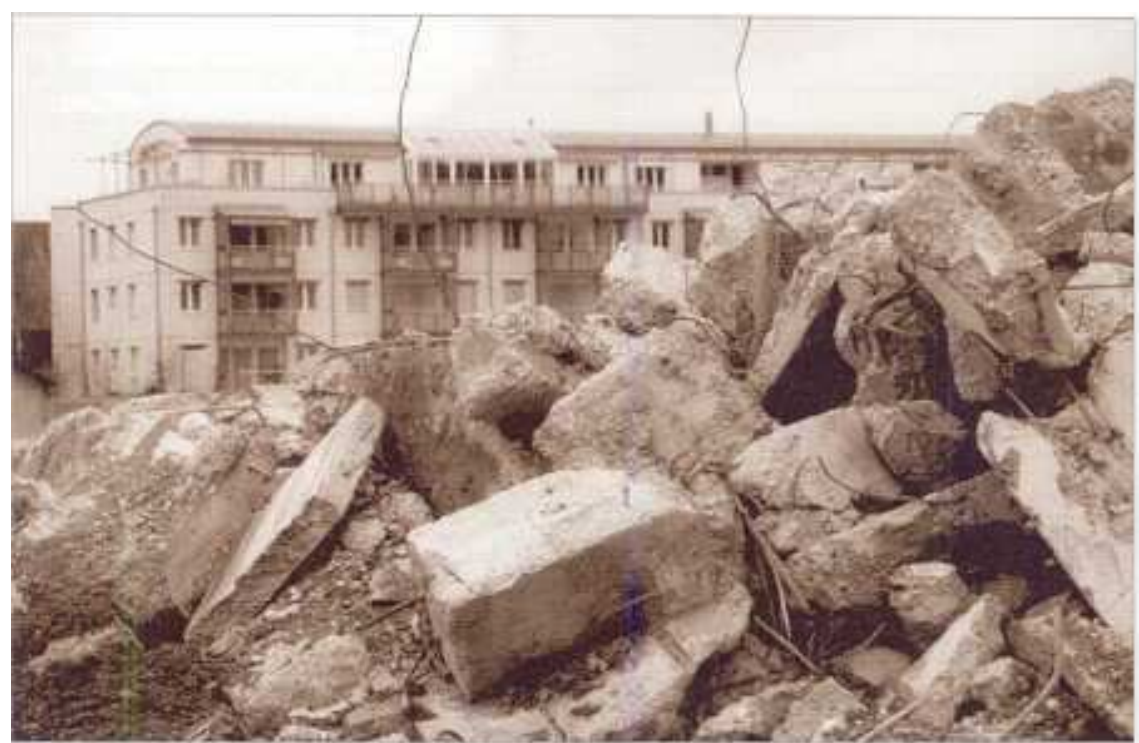

Bei einem Umbau des Wohngebäudeparks könnte die Wiederverwendung von Altbeton aus den abgebrochenen Gebäuden bis zur Hälfte des Ressourcenbedarfs für die Ersatzbauten einsparen. liegt der Energieverbrauch im Bereich von 30 Prozent der in der Schweiz heute "nachhaltig" produzierten Energie $(14000 \mathrm{MJ} / \mathrm{E}$ a Wasserkraft, $1700 \mathrm{MJ} / \mathrm{E}$ a Biomasse, $1200 \mathrm{MJ} / \mathrm{E}$ a Abfall). Die Haushalte könnten also - den gleichen Anteil am Gesamtenergieverbrauch wie heute vorausgesetzt - knapp "solar" betrieben werden.

\section{Folgerungen für den Ressourcenhaushalt in der Aktivität Wohnen}

Die drei vorgestellten Szenarios illustrieren die Spannweite von naturwissenschaftlich und verfahrenstechnisch gestützten Entwicklungsvarianten beim Bau zukünftiger Wohngebäude. Es versteht sich von selbst, daß der wirtschaftlichen Bedeutung des Wohnungsbaus mindestens das gleiche Gewicht zugemessen werden muß. So wurde bereits früh darauf hingewiesen ${ }^{[8]}$, daß der ökonomische Aufwand zur Werterhaltung des rasch gewachsenen "Lagers" oder "Parks" von Wohngebäuden zu einem Schlüsselproblem künftiger Generationen werden könnte. Eine rein wirtschaftliche Grobbeurteilung läßt also Zweifel an der "Nachhaltigkeit" gegenwärtiger Bauwerke und eines Szenarios 


\section{Gebäudevolumen}

\section{Energieverbrauch}

\section{Szenario "Business as usual"}
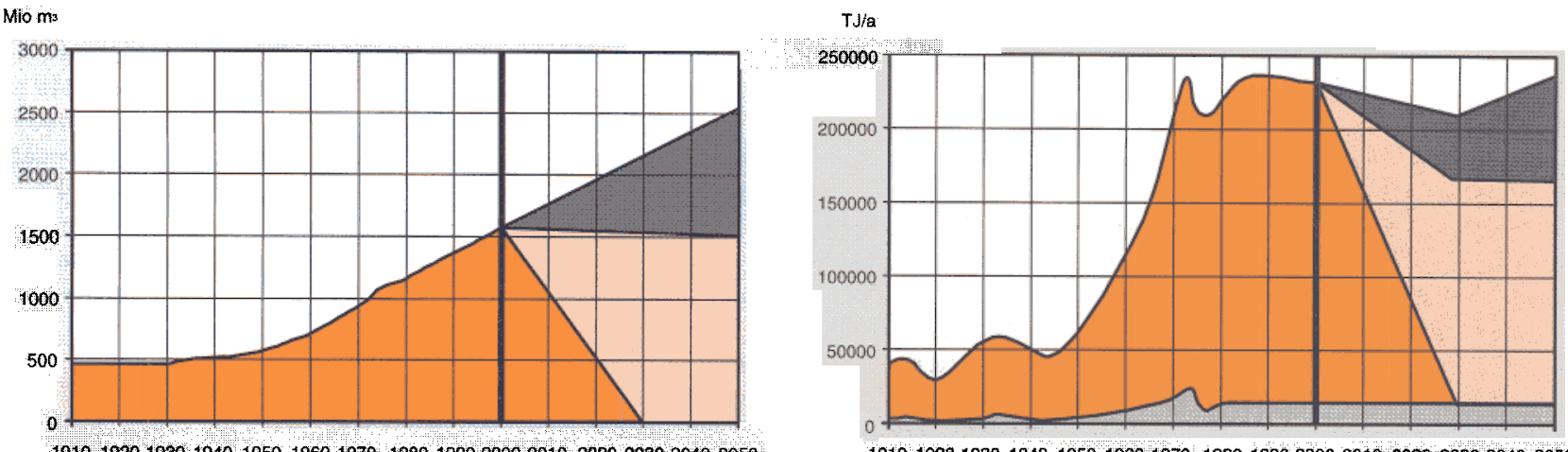

Jahr

\section{Szenario "Erhalten"}
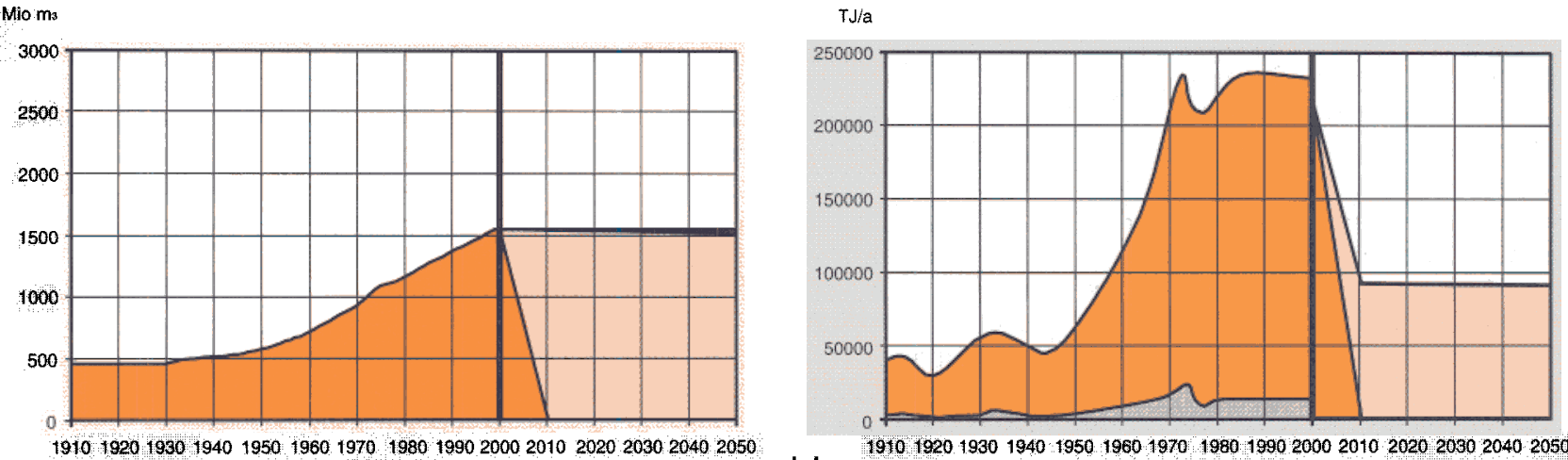
Jahr

\section{Szenario "Umbauen"}

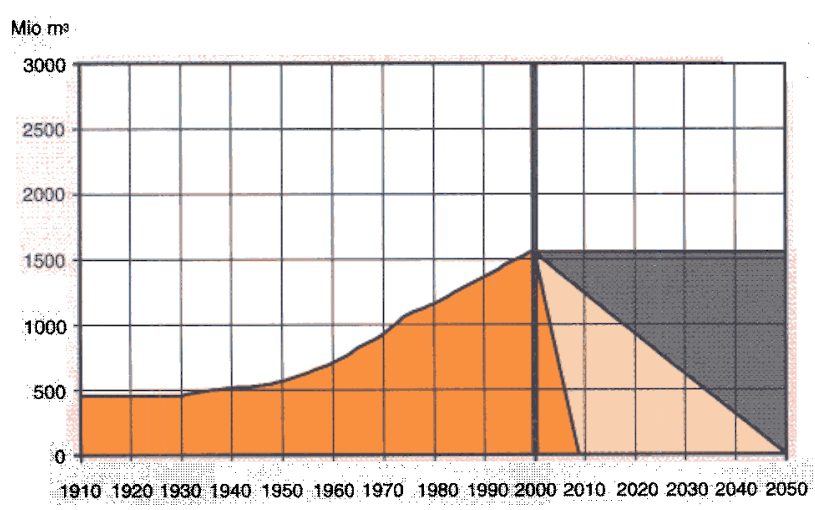

Gebäudevolumen "alte Wohngebäude" $V^{3}(t)$

Gebäudevolumen "sanierte Wohngebäude" $V^{4}(t)$

Gebäudevolumen "neue Wohngebäude" $V^{5}(t)$
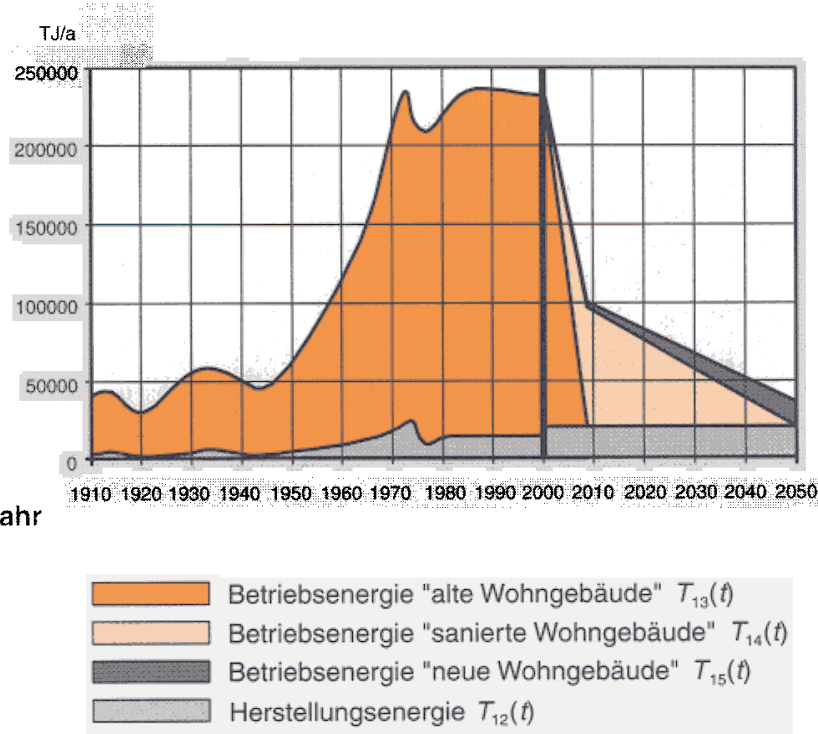

Figur 5. Entwicklung von Gebäudevolumen und Energieverbrauch bei den drei Szenarios. Versteht man Energieeffizienz als Energieverbrauch (Aufwand) pro Gebäudevolumen (Nutzen), so läßt sich erkennen, daß die Energieeffizienz beim Szenario "Umbau" am stärksten ansteigt. Beim Szenario "Business as usual" werden Energieeinsparungen durch Sanieren längerfristig durch das anhaltende Gebäudewachstum wieder geschluckt. 
"Business as usual" aufkommen [20]. Die metabolische Betrachtung verschafft einige zusätzliche Antworten auf die in Abschnitt 2 dieses Beitrags gestellten zwei Fragen und erlaubt darüber hinaus zwei Thesen zur "Nachhaltigkeit" in der Siedlungsentwicklung:

- Für die nächste Generation ist die eigentliche "Altlast" des in den vergangenen hundert Jahren aufgebauten Zwischenlagers von Wohngebäuden nicht dessen stoffliche Menge und Zusammensetzung, sondern der Energieaufwand für seinen Betrieb.

Das Setzen von einzelnen Nutzungszielen für eine nachhaltige Ressourcenwirtschaft kann ohne Beachtung der Systemeigenschaften kontraproduktiv sein. Im hier betrachteten Fall könnten zum Beispiel strikte Kiesverknappungen einen wesentlich effizienteren Energiehaushalt verhindern. Geht man zudem davon aus, daß für die Herstellung von Beton auch Bruchsteine verwendet werden könnten, deren Gewinnung relativ wenig zusätzliche Energie (gemessen am Gesamtaufwand, siehe Figur 4) braucht, so wird die Schonung der quartären Kieslager zweitrangig. Anders formuliert: Künftige Generationen gewinnen mehr Gestaltungsfreiheit, wenn der Energiebedarf für den Betrieb der Gebäude drastisch reduziert würde, als wenn man zuerst am Baumaterial Kies zu sparen suchte. Hingegen wird deutlich, wie wichtig die bestehenden und zukünftigen Wohngebäude als Ressourcenlager einzustufen sind. Sie werden heute aus ökonomischen Gründen noch

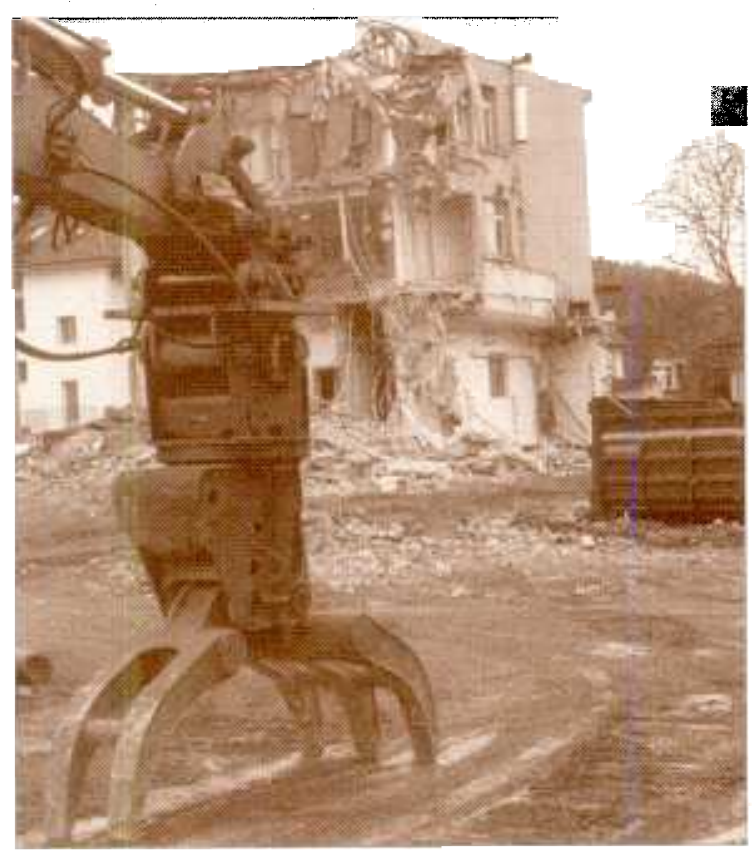

Umbauen heißt auch abbrechen:

Ein kontinuierlicher Ersatz von alten Wohngebäuden durch neue, besonders energieeffiziente Gebäude ermöglicht eine starke Reduktion des Energieverbrauchs. der Raumordnung und die Umsetzung der Raumplanung steuern. Das Studium solcher Vorgaben (zum Beispiel Grundzüge der Raumordnung Schweiz 1996) führt zum Schluß, daß sie die Verbindung zur Nachhaltigkeit fordern, aber eine konkrete Verknüpfung mit der Energiepolitik vermissen lassen. Mit anderen Worten: Es gilt auch der Umkehrsatz zur obengenannten These. Konzepte der Siedlungsentwicklung, die sich auf Nachhaltigkeitskriterien beziehen, sind nur dann tauglich, wenn sie sich an langfristigen Vorstellungen der Energiepolitik orientieren.

\section{Gesellschaftspolitische Prozesse in der Siedlungsentwicklung}

Eine ganze Reihe jüngster Publikationen weist darauf hin, daß am Ende des 20. Jahrhunderts die Frage der urbanen Entwicklung vielfach Ratlosigkeit begegnet. Einerseits werden die einstigen stolzen "Kernstädte" wie schwerkranke Patienten behandelt, deren Heilung fraglich ist. Anderseits gibt es im urbanen Netz einige Anthropotope, die in blendender Verfassung sind, aber von den leidenden Nachbarn als Parasiten angesehen werden. Der Begriff "Krise" führt zur Zeit die Liste der häufigsten Slogans an, wenn von Stadtentwicklung die Rede ist. Die Facetten dieser Krise reichen vom sozialen Zerfall über den wirtschaftlichen Niedergang bis zum architektonischen Chaos.

Mitunter lassen sich auch einige Querdenker vernehmen (zum Beispiel Koolhaas ${ }^{[23]}$ ), die das Jammern satt haben. Sie akzeptieren das bisher bewußt Geschaffene oder auch zufällig Entstandene ohne Mißmut und schöngeistiges Bedauern über verpaßte Gelegenheiten. Es ist das Material, mit dem wir zu arbeiten haben. Nichts anderes ist vorhanden. Es geht nicht darum, auf der grünen Wiese irgendwo die neue Stadt $\mathrm{zu}$ bauen. Vielmehr kommt es darauf an, die Städte, in denen wir leben, neu zu denken und sie zu verändern.

Ein solcher Umbauprozeß verlangt nicht nur professionelle Stadt- und Raumplaner und verordnete Konzepte "top down". In demokratischen Gesellschaften braucht es auch den Mut zu neuen gesellschaftspolitischen Experimenten, in denen Urbanität in unabhängigen Projekten "bottom up" erfunden wird. Aus dem Deutschen Institut für Urbanistik zum Beispiel kommt der Vorschlag ${ }^{[24]}$, auf die Herausforderung 


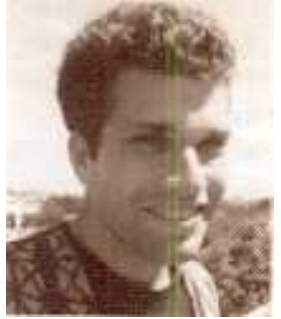

Michael Redle: Geboren 1966 in Basel. Studium der Umweltnaturwissenschaften an der ETH Zürich. Seit 1992 Assistent und wissenschaftlicher Mitarbeiter der Professur Stoffhaushalt und Entsorgungstechnik der ETH Zürich und der gleichnamigen Abteilung der EAWAG in Dübendorf. 1994-1998 Doktorarbeit in der Forschungsgruppe "Regionaler Stoffhaushalt" mit dem Thema "Ressourcenbewirtschaftung von Kies und Energie in Abhängigkeit von der Siedlungsentwicklung". Während dieser Zeit Mitarbeit im ETH-Forschungsprojekt "SYNOIKOS - Nachhaltigkeit und urbane Gestaltung im Raum Kreuzung Schweizer Mittelland" sowie im EU-Projekt "Material Accountig as a Tool for Decision Making in Environmental Policy".

Peter Baccini: Geboren 1939 in Zürich. Studium der Naturwissenschaften an der ETH Zürich und in den USA; Promotion in Chemie 1968 an der ETHZ. Fünfjährige Tätigkeit in Forschung und Entwicklung bei der chemischen Industrie in Basel (1968-1973). Gewässerforschung am Seenlaboratorium der EAWAG in Kastanienbaum, Luzern (1974-1983). Professeur associé für Umweltchemie an der Universität Neuchâtel (1974-1989). Gastwissenschaftler an der Universität Göttingen und an der University of California in Riverside (1981). Heute: Professor für Stoffhaushalt und Entsorgungstechnik an der ETH Zürich und Leiter der gleichnamigen Forschungsabteilung an der EAWAG in Dübendorf.

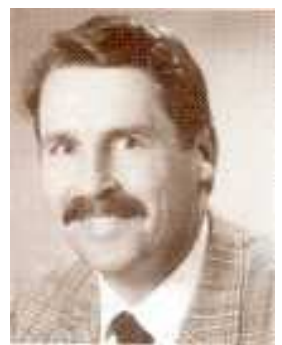

der Globalisierung mit dem Aufbau neuer regionaler Strukturen zu antworten. Vor allem soll die kommunale Ebene politisch gestärkt werden, damit die sozialen Integrationsarbeiten und der ökologische Stadtumbau gelingen. Demgegenüber steht die jüngste Erfahrung aus einer föderalistischen Region par excellence, dem Schweizer Mittelland ${ }^{[25]}$. Die aus dem 19. Jahrhundert stammenden politischen Strukturen wie Gemeinden, Bezirke, ja sogar Kantone bilden das Haupthindernis dafür, den längst als notwendig erkannten Umbauprozeß einer eng verflochtenen "Stadt Schweiz" mit rund 5 Millionen Köpfen in die Wege zu leiten. $\mathrm{Zu}$ hohe Gemeindeautonomie und zu kleine Einheiten können das Größere paralysieren.

Beide Annäherungen haben das gleiche Ziel: die erste mit der Motivation, der Globalisierungsfalle auszuweichen, die zweite mit der Absicht, sich aus den Fesseln der übermächtigen Gemeindeautonomie zu lösen. Das Ziel heißt, aus bestehenden Siedlungen unterschiedlicher Qualitäten "neues gutes Stadtleben" hervorzubringen. Und dazu braucht man ungewöhnliche gesellschaftspolitische Prozesse, die noch zu erfinden sind. Dieses Anliegen ist alt und erinnert an den in der "Stadttheorie" entstandenen Begriff des Synoikismus. Er umschreibt das in der Geschichte bereits über Jahrtausende bekannte Phänomen des Zusammenschließens von mehreren "Ortschaften" zu einem größeren "urbanen Organismus". Solcher Zusammenschluß ist ein höchst anspruchsvoller politischer Prozeß. Der
Schlüssel dazu liegt im "regionalpolitischen Willen" der Menschen, sich eine neue urbane Identität zu schaffen.

Für kritische Anmerkungen und Diskussionen danken wir Susanne Kytzia, Hans-Peter Bader und Georges Henseler, Abteilung Stoffhaushalt und Entsorgungstechnik, EAWAG.

\section{Literaturverzeichnis}

[1] M. Buitenkamp, H. Venner, T. Wams (Ed.): Action Plan Sustainable Netherlands, Friends of the Earth, P.O. Box 19199 Amsterdam (1992).

[2] P. Baccini, H.-P. Bader: Regionaler Stoffhaushalt - Erfassung, Bewertung und Steuerung, Spektrum Akademischer Verlag, Heidelberg (1996).

[3] P. Baccini: "Understanding Regional Metabolism for a Sustainable Development of Urban Systems". Environmental Science \& Pollution Research 3 (1996) 108-111.

[4] S. Kytzia, S. Friedrich, C. v. Fischer: "Gewohntes verändern - Leitfaden für den Entwurf von Umbauszenarien", in P. Baccini, F. Oswald (Ed.): Netzstadt Transdisziplinäre Methoden zum Umbau urbaner Systeme, vdf Hochschulverlag, Zürich (1998), p. 88-124.

[5] P. Baccini, F. Oswald (Ed.): Netzstadt Transdisziplinäre Methoden zum Umbau urbaner Systeme, vdf Hochschulverlag, Zürich (1998)

[6] Bundesamt für Statistik: Eidgenössische Volkszählung 1990, Gebäude und Wohnungen, Thematische Tabellen, BFS, Bern (1993).

[7] IP BAU: Dokumentation Gebäudebestand Schweiz, Bundesamt für Konjunkturfragen, Bern (1991).

[8] C. Gabathuler, H. Wüest: Bauliche Ressourcen und ihre Benützung, Lagebeurteilung und mögliche Veränderungspotentiale, ETH-Forschungsbericht MANTO,
Zwischenbericht 2, Spezialstudie 2.23, ETH Zürich (1984).

[9] Bundesamt für Statistik: Bau- und Wohnbaustatistik der Schweiz, Bauinvestitionen und -vorhaben 1994-95, Wohnbautätigkeit 1994, BFS, Bern (1996)

[10] Verein Schweizerischer Zement-, Kalkund Gips-Fabrikanten: Einführung in die schweizerische Zementindustrie, Zürich (1986).

[11] C. Siegenthaler, H.C. Binswanger: Lenkung des kantonalen Kies- (und Kiesersatz-) Abbaus durch planerische Massnahmen oder Abgaben aus der Sicht einer ökologisch-ökonomischen Gesamtbilanz, Studie im Auftrag des Solothurner Baudepartements, Institut für Wirtschaft und Ökologie, Hochschule St. Gallen (1993).

[12] D. Notter: "Comtabilité énergétique globale de trois bâtiments", Sonnenenergie 3 (1996) 34-36.

[13] Laboratorium für Energiesysteme, ETH Zürich/PSI Villigen, Forschungsbereich 4: Ökoinventar für Energiesysteme (1994)

[14] B. Wick: "Energiekennzahlen - Neuere Erkenntnisse über die Kennzahlen", Schweizer Ingenieur und Architekt 38 (1991) 893-896.

[15] "Schweizerische Gesamtenergiestatistik 1992", Sonderdruck aus Bulletin SEV/VSE, 12 (1993).

[16] B. Wick: "Energie im Mehrfamilienhaus: Verbrauchswerte und Sparpotential", Schweizer Ingenieur und Architekt 5 (1982) 50-56.

[17] O. Humm: "Niedrigenergie-Siedlung Boller in Wädenswil ZH", Schweizer Ingenieur und Architekt 7 (1996) 4-8.

[18] A.R. Keller: "Der Blick in die solare Zukunft", Sonnenenergie 6 (1995) 12-15.

[19] T.C. Hansen: "Recycled Aggregates and Recycled Aggregate Concrete", in Recycling of Demolished Concrete and Masonry, RILEM Report 6, E \& FN Spon, London (1992)

[20] S. Friedrich, S. Kytzia, C. v. Fischer, F. Oswald P. Baccini: "Umbau des Wohnens - Werkstattbericht aus einem transdisziplinären Forschungsprojekt", DISP 129 (1997) 23-29, Institut für Orts-, Regional- und Landesplanung, ETH Zürich.

[21] D. Müller: "Szenarien zur nachhaltigen regionalen Holzbewirtschaftung", Schweizerische Zeitschrift für Forstwesen 147/11 (1996) 873-885.

[22] C. Blaser, M. Redle: "Mehr Mobilität mit weniger Verkehr - Umbauszenarien zur Aktivität Transportieren und Kommunizieren", in P. Baccini, F. Oswald (Ed.): Netzstadt - Transdisziplinäre Methoden zum Umbau urbaner Systeme, vdf Hochschulverlag, Zürich (1998), p. 126-153.

[23] R. Koolhaas: "Whatever Happened to Urbanism?", in R. Koolhaas, B. Mau (Ed.): Small, Medium, Large, Extra-Large: Office for Metropolitan Architecture, 010 Publishers, Rotterdam (1995)

[24] H. Mäding: "Entwicklungsperspektiven für die Stadt - Trends und Chancen", Aktuelle Information (Dezember 1997), Deutsches Institut für Urbanistik, Berlin.

[25] P. Baccini, G. Dürrenberger, S. Friedrich, F. Oswald: Stadt an der Wigger - Impulse für die Zukunft setzen, Professur für Architektur und Städtebau / Professur für Stoffhaushalt und Entsorgungstechnik, ETH Zürich (1998).

(Eingegangen am 10. Juli 1997) 